\section{(Clinital Ieturte}

\section{ON THE \\ SURGICAL TREATMENT OF HYDATID TUMOURS IN THE ABDOMEN.}

Delivered at the London Hospital.

BY

JONATHAN HUTCHINSON, F.R.C.S.,

SURGEON TO THE HOSPITAL, AND LECTURER ON SURGERY IN THE MEDICAL SCHOOL.

Genthemen,-We discharged a few months ago from Talbot ward, a man whose case had excited much interest, as an instance of a large hydatid tumour in the abdomen cured by operation.

He was a man aged about 45. You may recollect that he was admitted on account of a large fluctuating tumour, which presented immediately beneath the edge of his lower ribs in the left dorsal region. He had been aware of the existence of a tumour in this part for several months, and latterly it had occasioned him much pain. He still, however retained good general health. When, in the first instance, he applied as an out-patient, my colleague, Mr. Little, introduced a grooved needle into the swelling, with the result of obtaining a small quantity of clear fluid. The knowledge of this fact much helped our diagnosis when the man came under our care in the ward. We had to deal with a large swelling which filled the upper part of the lumbar region, which received an impulse on coughing, which fluctuated distinctly, and from which a clear fluid had been obtained by exploratory puncture. The man had no symptoms of disease of the spine; nor were there the physical signs of pleural effusion, nor the usual history of pleurisy. Under these circumstances, our conjecture was, that the tumour was probably of hydatid nature. The pain which the man had recently suffered made him very willing that we should adopt any means thought best. A few days after his admission, I made an opening into the tumour in the following manner. An incision having been made through the skin, fascia, etc., the tumour was well held backwards, and I then thrust a narrow bladed knife deeply into the most prominent part.* This was followed by a gush of watery fluid, slightly turbid, but still very different in appearance from any form of pus. After the knife, I passed a pair of dressing forceps, by which the deep puncture was stretched until it admitted a piece of India-rubber tubing, which latter was secured in position in the usual manner. At least a pint of fluid had escaped from the puncture, and it continued afterwards to run away freely through the tube. As nothing but fluid had been seen, and as that fluid was necessarily mixed with blood from the wound, I was unable to speak positively as to its coming from the interior of a hydatid cyst; but I stated my belief that it did so.

The man was feverish and rather ill for several days after the operation. The fluid became turbid and purulent, and was secreted in large quantity. About ten days later, we removed the tube, and enlarged the fistula by introducing a pair of long narrow forceps and then opening the blades.

* In this case, I preferred to open the tumour with the knife instead of using the trocar, for several reasons. 1. The diagnosis was not certain. 2. The tumour was probably belind the peritoneum. 3. I thought it probable that it had already inflamed, owing to the previous puncture On reviering the that a trocar evacuation would have been the best practice.
A few days after this, our diagnosis was made certain by the escape of a fragment of white leathery membrane, about an inch square, semi-transparent, and resembling a lamella of boiled onion. This fragment I now show you. There cannot be felt the least doubt as to its nature by any one who has ever seen the like before. If, however, any doubt could be felt, it is at oncedispelled by the inspection of a section under the microscope. You see that it is made up of closely packed concentric lamellæ like the layers of an onion, or, still better, like the lines of a fortification agate. No structure, as far as I am aware, found in the human body presents this peculiar appearance excepting the hydatid membrane.

After this fragment had escaped, I repeatedly stretched the fistula by forceps with the view to facilitate the escape of others, and great quantities came away. On one occasion, I was shown a porringer full of these pieces of membrane, which had been forced out during a coughing fit on the previous evening. All were broken and in a state of decomposition, the pus in which they floated stinking horribly. With the microscope, I detected echinococci and their detached hooklets in considerable numbers.

The man was now much improving in health. $\mathrm{He}$ had a good appetite, and little or no pain. About two months after his admission, we allowed him to return home, as he was very anxious to go to work. No portions of hydatid membrane had escaped for a fortnight prior to his discharge, and the secretion of pus had indeed almost ceased, its fœtor being quite lost. I had taken care to keep the external orifice well open; and my belief is that the cyst was quite emptied of its parasites, and that the fistula would very shortly heal.

With regard to the exact structure in which the cyst was in this case developed, I can give no positive opinion. We had no evidence that it had any connection with the lungs, liver, kidney, or spleen and my impression is, that it had probably found its bed in the subperitoneal cellular tissue.

A fortnight ago, we admitted another case, in which, although as yet we have not confirmed the opinion by the result of a puncture, yet I venture a confident diagnosis that the tumour is hydatid.

Alice Stiff, the child now before us, is, as you see, a little girl having the appearance of perfect health, and about four years old. She was brought to the hospital on account of a hard swelling in her abdomen, of the existence of which her parents had only become aware a few weeks previously. It has occasioned no symptoms whatever.

The tumour rises as a very visible rounded swelling to the right of the navel, extending both above and below it. It is freely movable, and no pain is caused by handling it. In its centre is an obscure sense of fluctuation; but at its sides it is very firm. Different opinions have been given confidently by those who have examined it as to whether or not fluctuation is really present. I cannot myself feel any doubt about it. On the left side, the free edge of the liver can be easily felt continuous with the globular. enlargement ; and here also an oblique notch extending upwards and to the right can be distinguished (fissure between the right and left lobes). There is no pushing out of the ribs, from which fact it seems probable that the liver is dragged downwards by the weight of the tumour developed in its structure. The size of the tumour is, perhaps, about that of two fists. The tumour is so movable that when the child is made to lie on her left side it falls over towards the left. It is quite clear that there are no peritoneal adhesions. On percussing from above downwards, it is 
found that where the lung clearness ends there the hepatic dulness begins, and that the latter is continuous with the tumour. There is no enlargement of the spleen. I cannot detect hydatid fremitus with any degree of certainty.

I have ventured the diagnosis of an hydatid cyst with much confidence. If we proceed by the method of exclusion, it is almost impossible to suggest any other which should be at all plausible. It is true, that hydatid tumours have been very rarely indeed met with at so early an age ; but there is no $a$ priori reason why they should not occur. The diagnosis being, therefore, considered to be established, we come to the important question as to what treatment is advisable. We have the choice between two plans only. Either we may let it alone, and trust to natural processes; or we may operate with the view of procuring the death of the parasite and the contraction of the cyst. No known medicine affords the slightest chance of advantage.

Now, gentlemen, I will invite you to feel very incredulous as to the necessity of any interference in the case before us. The little patient is in good health and suffers no inconvenience from the tumour; why subject her to the risk attending a trocar-puncture into the substance of her liver? I reply, that it is probable in a high degree that the tumour will increase in size ; that it will before very long become the cause of pain and of disturbance to the functions of the adjacent organs; and that eventually it will jeopardise life. I allege, on the other hand, that the operation of puncture is attended by very little risk ; and that it is highly probable that it will effect a permanent cure. Let me produce evidence on these points.

I will first relate one of the most satisfactory cases which has occurred in the whole of my surgical experience. Last winter, I was requested by Mr. Marsh, of St. John Street, to see a patient of his who was suffering from an abdominal tumour of a doubtful nature.

Mrs. B., aged about 33, married, and the mother of a family, had only quite recently come under $\mathbf{M r}$. Marsh's care. She had previously been seen by several other surgeons, by whom, in the belief that the disease was malignant disease of the liver, a most unfavourable prognosis had been given. She was indeed very ill; and, at first sight, there seemed much to confirm this view of the case. She had lost her flesh greatly, and was of pale sallow complexion. The suspicion that the tumour might prove to be hydatid and not malignant had, however, occurred both to Mr. Marsh and to Dr. Hughlings Jackson, who had examined it before I was called in When we met in consultation, we found Mrs. B. much emaciated, quite confined to her bed, and suffering from almost constant sickness. It had been necessary to give morphia freely, in order to procure rest and relief from pain. The tumour filled the epigastrium, from the lower part of the sternum to the umbilicus, and extended laterally into the hypochondriac region on each side, lifting the lower ribs. On the sides it felt very firm indeed; but at the epigastrium we thought that fluctuation could be detected. The bulging of the whole region was quite perceptible to the eye. The integuments over the tumour were thickened and somewhat inflamed; but this seemed accidental, and connected rather with some boils which had formed than with the tumour.

The history was obscure. During the last few years, the patient had had several illnesses with abdominal pain ; but we made out satisfactorily that she had had neither dysentery nor jaundice. She had known of the existence of a tumour for about a year, and it had much increased of late. The sense of fluctuation obtained was that of fluid contained in an exceedingly tense cyst; and I thought I could elicit a certain degree of vibration (hydatic fremitus). We all agreed that the tumour was probably hydatid; but, partly because of the patient's dread of an operation, it was decided to defer any radical measures of treatment.

I saw her twice during the next ten days, and on each occasion my opinion was strengthened. At length, the patient's sickness exhausted her so much, that it became clear that we were running great risk in any longer deferring an operation. I accordingly punctured the tumour at about the middle of the epigas. trium with a small exploring trocar; and having obtained clear fluid, changed the instrument for one about the size of No. 4 catheter. With the latter, we drew off more than two pints of clear salt water. The fluid had not the slightest opacity ; and on subsequent examination, it was found to contain a large quantity of chloride of sodium and no albumen. There could be no doubt that it came from a hydatid cyst. The puncture was closed with lint and strapping.

After the tapping above described, the patient progressed uninterruptedly well; her sickness ceased; she took food well; and the tumour did not refill. We had, at the time, not nearly emptied it; we only drew off as much fluid as would easily flow. The cyst, however, did not inflame; at least, not so as to cause any constitutional symptoms. After a while, the fluid which had been allowed to remain was absorbed, and the cyst contracted.

Three months after the operation, the patient called upon me at my house. She said she was in as good health as ever she had been in her life, and her aspect fully confirmed her statement. There was scarcely any evidence of enlargement or thickening in her liver.

In this case, we have an instance in which a large hydatid tumour perished in consequence of a simple trocar evacuation of its fluid contents, and in which shrinking of the cyst occurred without inflammatory symptoms. The obstinate sickness from which the patient had suffered, was no doubt due to the mechanical pressure of the tumour on the stomach.

A case somewhat similar to this was under my care in the Metropolitan Free Hospital, in conjunction with my colleague, Dr. James Jones, about four years ago.

Our patient was an Irishwoman, aged about 36, and in fair health. Her tumour was very large; but it was developed in the right lobe of the liver, and had dragged that organ low in the abdomen instead of pressing upward, as in the previous case.

The patient was thin; and the boundaries of the tumour could be easily made out. There was no doubt as to its being a large tense-walled cyst. A certain degree of fremitus or vibration was discoverable. The lower ribs on the right side were lifted by the tumour, which also extended downwards nearly to the crest of the ilium. No doubt was felt by any of those who examined the case, as to the nature of the tumour. It had caused the patient much pain, and was increasing rapidly; so that there appeared much reason to apprehend that it might give way into the peritoneal cavity. It was accordingly determined to puncture it. This I did with a trocar of small size (about No. 3), selecting the most prominent part of the tumour. Fluid as clear as water and very salt escaped freely. We drew off about three pints, which had the effect of reducing the tumour to a third of 
its original size. The puncture wound was protected by lint and plaster.

During the week following the operation, the patient had rather sharp febrile symptoms, with tenderness over the tumour, either from peritonitis on its exterior, or from inflammation of the cyst itself. The latter became almost as large as it had been before, and contained a large quantity of air; over the whole of its front part it was tympanitic. That the air had been excreted, or was the product of decomposition, was certain; as none had been admitted at the time of puncture. In a short time, these symptoms passed off satisfactorily; and the woman made a good recovery, the tumour shrinking till it could scarcely be detected. She left the hospital well about six weeks after her admission. I saw her again some months later, and she was then wholly free from her disease.

I must ask you to note that, in this as in the preceding case, none of the smaller cysts were removed, and that the tumour shrank up in consequence of the evacuation of its contained fluid only. It is singular, that so simple a measure as a puncture with a small trocar should suffice to destroy the vitality of these parasites. We might have supposed that the fluid would be quickly re-secreted, and that their life and growth would continue as before. But it is not so; the withdrawal of the fluid in which they float suffices, I believe, always to accomplish their death. Their death may or may not cause inflammation of the part in which they are embedded.

Having thus brought before you the particulars of three cases in which excellent results were obtained by operative interference, I have now to mention one in which spontaneous recovery (partial) took place.

A young Irishman, of about 18, came under my care as an out-patient, about ten years ago, on account of a large tumour in the right lobe of his liver. The tumour occupied the whole right half of his abdomen, bulging out his liver, and displacing his ribs upwards. It fluctuated, and had the tense elasticity of an hydatid cyst. Excepting from the pain caused, the man was in good health; and despite its weight and inconvenience, he still kept at his work. I felt no doubt whatever as to the diagnosis. I advised him to come into the hospital, and have the tumour punctured; but this he refused. He remained under observation several months. At one time the tumour caused him so much pain, that he was obliged to give up his work. One morning, his friends sent, begging that I would come and see him, as they thought him dying. He had been unable to lie down all night; and the pain he suffered was such that I much feared the cyst would burst. I took a trocar with me; but again his courage failed, and he would not allow me to puncture it. From this time he improved. The attacks of pain became less frequent; and in a week or two he returned to work. I examined him six months later, and found the tumour diminished to half its original size, and quite free from pain. He promised to let me see him from time to time, but neglected to do so; and I have since not succeeded in obtaining any information respecting him. I believe he recovered, and that the cyst was in process of contraction. Let us clearly remember, however, that he ran the narrowest possible risk of his life. The tumour, when at its largest, probably contained not less than from a gallon to a gallon and a half of fluid; and it was a mere chance that it did not give way into the peritoneal sac.

The spontaneous death of hydatids is not a rare event; and when they do die, I believe, the cyst usually contracts, its fluid contents being absorbed, and no injury is caused to its possessor. At any rate, we not unfrequently, in the post mortem theatre, meet with specimens of collapsed and dried-up hydatids in the livers of those who have died of other disease. Here is one which Mr. Spencer removed a few months ago from a patient of mine who had died after injury to the head. The whole tumour is not larger than a hen's egg. It is lodged in the left lobe of the liver near its free margin. Closely adherent to the hepatic tissue, is a dense fibrous capsule secreted by the liver in consequence of the irritation of the parasite. Within that we have the parent hydatid cyst, much involuted and folded. The parent contains many smaller ones, all of them collapsed and shrunken. All are opaque and somewhat softened, and amongst them is a glutinous material with opaque yellow cheesy matter. Probably this specimen has been dead a few years. An almos7 exactly similar one, but three times as large, and shewing less evidence of decomposition, was removed two or three years ago from the liver of a patient of mine, who had died after strangulated umbilical hernia. It has chanced that I have three times met with these tumours in the autopsies of hernia patients ; and it suggests itself as possible, that the distension of the abdomen by them, when living and of considerable bulk, may possibly increase the risk of the occurrence of hernial protrusions. If you come upon a hydatid tumour at a still later period of degeneration after death than the one before us, you will find a dense cyst containing cretaceous and cheesy material without any evidence of secondary cysts whatever. In this chalky material, you will still often be able to discover with the microscope the hooklets of the echinococci.

But, although we often find post mortem proof that these parasites may die spontaneously, and cease to be the source of danger to their possessors, yet I believe we have but little reason to think that this fortunate event can be hoped for in cases in which the tumour has once attained a large size. Spontaneous death is probably brought about by external compression by the fibrous capsule which surrounds them; but this pressure must become very inconsiderable when they have reached a certain bulk. I know of no case on record parallel to the one I have mentioned, in which a very large one spontaneously diminished and probably died. There are, on the other hand, abundant cases on record in which these large tumours have caused death. You have but to turn to Dr. Bright's admirable Memoirs on Abdominal Tumours, or to Frerichs's excellent account of hydatid tumours given in his work on Diseases of the Liver, for instances of this. One such, which occurred at this hospital about two years ago, will be in the recollection of some of you. The subject of the latter was a man under the care of my colleague Dr. Little. The tumour was very large indeed ; and had displaced the diaphragm upwards, so as greatly to compress the left lung. A perfectly correct diagnosis had been given, and an attempt to puncture the cyst had been made; but, unfortunately, the trocar entered at a part where the livertissue was thick, and no fluid was obtained. It was intended to try again in a few days; but, meanwhile, the man rather unexpectedly died. The puncture had nothing to do with his death, which 
resulted, I believe, simply from the pressure of an immense cyst upon the thoracic contents.

The specimen obtained in Dr. Little's case was remarkable in that, although so very large, it contained no secondary cysts. You may, perhaps, be aware that the peculiar symptom known as hydatic fremitus is not met with if there be no floating cysts in the interior of the parent one. It consists in a kind of trembling of the cyst-wall felt by the hand some seconds after the ribrations produced by percussion have subsided; and is supposed to be produced by the secondary cysts which float in the interior bobbing against the wall of the parent, and thus causing a sort of percussion from within.

The drawing which I now show (see woodcut) was taken from echinococci obtained from the lining membrane of the cyst in Dr. Little's case. They were not numerous, and all that I found were dead. The sketch represents the body of one echinococcus and part of another; both are attached to the cyst-membrane. In both, the hooklets are all detached and floating loose in the body of the parasite. There are also seen crystals of some fatty matter-no doubt the product of decomposition of the dead animal. Had it not been dead, the hooklets would have been seen arranged in an orderly circlet on the animal's head.

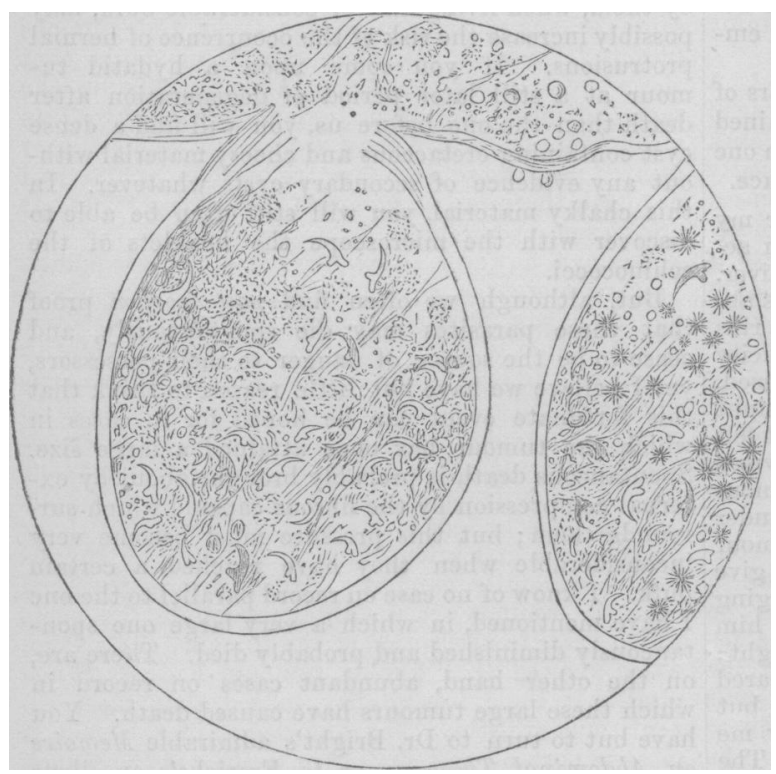

the practice of other surgeons, several other cases; in all that $I$ can recollect to have witnessed the result was good.

Of those recorded, in a certain number death has followed, either from escape of fluid into the peritoneum, or from the exhaustion and irritative fever consequent on the suppuration induced. Ill consequences have, however, been decidedly exceptional; and my impression is, that they will become still more so when is generally recognised what $I$ have endeavoured to show; viz., that simple puncture and evacuation of the fluid is all that is needed. In many cases, or indeed in almost all, the endeavour of the surgeon has been to secure a fistulous opening by which the secondary cysts might escape. With this object in view, various preliminary measures have been thought needful, to secure that the cyst should be adherent to the peritoneum before it was opened. Caustic potass and acupuncture, with incisions through the skin, only have, with this object, been tried. Then, again, in other cases, the patient's risk has been increased by the injection of irritant fluids, iodine, gall, etc., into the cyst, in order to destroy the parasite. If further experience shall prove that a simple puncture with a fine trocar is all that is needful, the treatment will have been rendered much more safe. I should recommend the use of a very fine exploring trocar-the fluid is quite thin, and will run easily through the smallest bore; and that no pressure should be made in the tumour, just so much fluid being allowed to escape as will run away spontaneously. The operator should have at hand a fine probe or wire with which to push away any floating cysts which chance to fall against the end of the cannula and stop the flow.

Occasionally, hydatid tumours open spontaneously, and are cured by the discharge of their contents. This, however, is a mode of termination attended by much inconvenience and often by danger. I have several times seen patients who had voided fragments of hydatid cysts with their urine, and in whom frequently symptoms of obstruction had been caused. A man who was in the habit of passing hydatids per urethram was, about a year ago, under Mr. Curling's care in this hospital. He suffered but little, and eventually quiterecovered. Sometimes, hydatids in the liver open through the diaphragm into the lungs; or, in other cases, into the stomach or intestine. Here is a fragment of hydatid cyst which a patient passed by the bowel; but which is so stained with bile that there can be no doubt as to whence

You will note the peculiar form of these hooklets, and their close resemblance to crabs' claws. It is desirable to have a trained eye for these little bodies, since their discovery in pus will often help out a diagnosis. They are remarkably difficult of destruction, and are to be found much more often in the pus discharged from suppurating hydatid cysts than are whole echinococci. Their form is so definite that, when once recognised, it is impossible to mistake them.

You may find, in the works I have mentioned, and also in many others, numerous cases in which large hydatid tumours have been punctured or opened with success. In addition to those which $I$ have cited, and which occurred to myself, I have seen, in it came. When the cyst obtains access to the pleura or lung, very serious symptoms may ensue. At the best, there will in such a case, in all probability, be prolonged fœtid expectoration, and the patient's strength will be much damaged.

Looking, therefore, candidly, at all the aspects of the question-1, the improbability that large cysts will be cured spontaneously; 2 , the dangers which attend them in respect to possible rupture into the peritoneum, or the effects of pressure on adjacent viscera; 3 , the inconvenience and risk incurred should spontaneous evacuation take place; 4 , the very little danger that attends a simple puncture; and, lastly, the great probability that such puncture will be sufficient to produce a cure-we may, 
I think, safely conclude that, when hydatid tumours in the abdomen have attained a size large enough to be recognised as such, it is the best practice to puncture them.

\section{Apettsomian aldectures \\ ON}

\section{MIDWIFERY AND DISEASES OF WOMEN.}

Delivered before the Medical Society of London. BY

C. H. F. ROUTH, M.D.,

PHYSICIAN TO THE SAMARITAN hOSPITAL FOR WOMEN AND CHILDREN.

\section{LeCture I. (Continued.)}

Or Some Points connected with the Pathology aNd Etiology of Fibrous Tumours OF THE UTERUS.

2. Fibroids are heterologous growths, which may be described as originating from an abnormal or pathological blastema, and they are of two kindsthe fibro-plastic and fibro-nucleated, each variety being a permanent pathological state, resembling one of the transient forms of fibrous tissue.

$a$. One way in which fibrous tissue grows is-1. A blastema appears; 2 . In this are formed a number of nuclei : these become cells with a single nucleolus. The cells elongate when split at the ends, and finally fibres are found. Lastly, the nucleoli disappear. The cells during elongation come to be spindleshaped. Now the fibro-plastic tumour of Lebert is a tumour made up of these bodies, the nuclei being called fibro-plastic nuclei, the spindle-shaped cells fusiform bodies, and the fibres fibro-plastic fibres. In a fibrillary tumour these several bodies exist in vast numbers. The cells are perhaps more rounded than in ordinary fibrous tissue: the fibres sometimes exist in very large numbers, so that the tumour may be said to be almost entirely made up of them. The nuclei, however, never entirely disappear.

$b$. The fibro-nucleated fibroid is a permanent condition of the second mode of growth observed in fibrous tissue. In this tissue the fibres may be formed directly from the blastema without the intermediate agency of cells, $i$. e., directly from the blastema; so that we have an appearance of cells and fibres quite distinct from one another. There may sometimes be very few fibres, so that the tumour may be said to be made up almost entirely of cells. Commonly, however, the fibres are present. Fibro-nucleated tumours are generally more malignant, and not necessarily encysted or circumscribed.

Intermediate between these two kinds of fibroids we may have very numerous differences, so that it may almost be difficult to say to which a given tumour exists, although the broad characters of a fibroid exist. To some such modification the recurrent fibroid may be referred, which is occasionally found in the uterus, in fibrous polypi, and in pelvian bodies. From the researches of Paget, which have been confirmed by subsequent writers, these tumours resemble the common fibrous tumours which are whitest, most homogeneous, least fasciculated and glistening. Microscopically, the cells are more elongated, caudate, oat-shaped, many with long and subdivided terminal ends. Nuclei and grumous and granular matter, such as might arise from disintegrated cells, are also present. The fibres are very few and far between.

3. Malignant fibroids are said by Paget to resemble exactly in all particulars the ordinary fibrous tumours of the uterus, with undulating white bands. The whole substance of the tumour has the same characters microscopical and general as these, although repeatedly examined, i. e., nothing but the compact well-formed fibrous tissue with imbedded elongated nuclei. On boiling, gelatine is freely yielded.

The association of cancer and fibrous tumours has been denied. The later pathological records, however, seem to leave no doubt as to their co-existence. Mr. Hilton exhibited to the Pathological Society (Trans., xii, 219) a tumour of a cancerous nature probably, following a recurrent fibroid. M. Bouvier (Annales de Chirurgie, iv, 21) gives a case of similar atnure, quoting others from Cruveilhier and Dumas. A similar case is given in the Bulletin de la Soc. Anat. (for April 1861, case 7), by M. Gillet, of general cancer in the abdominal organs with fibrous bodies in utero undergoing cancerous transformation. In a case to which I shall refer in the sequel, in the same tumour portions were cancerous and portions fibrous. To my mind, there can be no doubt that these two diseases do not uncommonly exist in the same tumour; and if malignant be understood to mean a tumour which may recur, and when cut or injured may leave an ulcerated surface which will not heal, there can be no doubt that this state is not uncommon in fibroids.

I cannot, however, stop here to discuss these points. To give, moreover, a treatise on fibrous tumours is no part of my intention. I can only glance at a few points, which in the sequel may be found useful to illustrate the practical points to which I wish particularly to call your attention. Whether we have to do with a true fibroid or fibroma with superimposed muscular tissue, these new growths in one important respect differ from uterus or muscle. If a fibrous tumour be wounded, so far as I am aware, it never heals by the first intention; it is followed by suppuration, generally gangrene. In a few exceptional cases the wounds set up an irritation in the parts which leads to absorption of the tumour. Years ago, Atlee showed that fibrous tumours had very little vitality in them, less vitality, indeed, than most adventitious growths. The knowledge of this fact is most important, as I shall hope to show in the sequel. For, while advantage has been taken of it to perform certain kinds of operations upon fibrous tumour, as in enucleation, it has been completely overlooked in the operation of gastrotomy, and has been often, I believe, a cause of death.

The position which fibrous tumours occupy in re. lation to the uterus has led to their subdivision into three classes, each of which has peculiar symptoms of its own. 1. Extrauterine or surface tumours, $i$.e., where they are situated just beneath the peritoneum. 2. Intrauterine or cavity tumours, where they are within the cavity of the uterus. 3. Intramural or parietal tumours, where they are imbedded in the walls of the organ. If the tumour-points be intermixed with much uterine tissue interposed here and there, some have made this another variety, and 